\title{
INFLUÊNCIA DA FLUTUAÇÃO CAMBIAL NO VOLUME DE VENDA DE AERONAVES: ESTUDO DE CASO DA EMBRAER
}

\author{
S.M. Vieira ${ }^{1}$; T.A.P. Linke ${ }^{1}$; M.V. Nascimento ${ }^{1}$ \\ 1 Faculdade de Tecnologia de São José dos Campos - Professor Jessen Vidal \\ Av. Cesare Mansueto Giulio Lattes, 1350 - Eugênio de Melo, São José dos Campos/SP, \\ CEP.: 12247-014, Brasil. \\ Telefone: (12) 3905-2423. \\ *tathianeps@gmail.com
}

\begin{abstract}
RESUMO: A EMBRAER emprega atualmente, cerca de 16.000 colaboradores no âmbito nacional, sendo o seu produto, o item industrializado mais exportado do país. Este artigo tem o objetivo de analisar a influência das flutuações cambiais na venda de aeronaves da referida empresa. Foram feitas pesquisas bibliográficas e desenvolvidos modelos matemáticos de regressão linear com dados coletados da empresa em estudo. Os dados foram coletados no período de 2015 até 2018 de forma trimestral. Esses dados são de domínio público e divulgados pela própria empresa. Apesar do modelo indicar que há uma relação positiva entre a cotação cambial e a venda de aeronaves, a análise estatística do modelo invalida qualquer possibilidade de real impacto da flutuação cambial no volume de vendas de aeronaves. Por outro lado, foi gerado outro modelo matemático no trabalho que indica que cada aeronave vendida potencializa em 112 milhões de reais a receita líquida da empresa.
\end{abstract}

PALAVRAS-CHAVE: flutuação cambial; regressão; venda de aeronaves.

ABSTRACT: EMBRAER currently employs around 16,000 employees nationwide and its product is the most exported industrialized item in Brazil. The purpose of this article is to analyse the influence of exchange rate fluctuations on the sale of aircraft by EMBRAER. To accomplish this objective this paper presents bibliographical researches and mathematic models of linear regression with data collected from the period between 2015 and 2018 on a quarterly basis. This data is public domain and disclosed by the company itself. Although the model indicates that there is a positive relationship between the exchange rate and the sale of aircraft, the statistical analysis of the model invalidates any possibility of a real impact of the exchange rate fluctuation on the aircraft sales volume. On the other hand, it was generated another mathematical model in the work that indicates that each aircraft sold increases in 112 million reais the net revenue of the company

KEYWORDS: exchange rate; regression; aircraft sales.

\section{INTRODUÇÃO.}

Segundo Williamson (2003), o desenvolvimento baseado em taxas cambiais é a principal variável de política econômica em um país, ou seja, para motivar a produção de manufaturados para exportação, gerar mais investimentos, empregos e crescimento econômico, é preciso uma taxa cambial competitiva.

Esse potencial de produção para exportação é explicado pela teoria de economia clássica que segundo Giannetti (2005) existe correlação positiva entre a flutuação do câmbio e o desempenho do exportador, uma vez que, quanto maior a depreciação da moeda nacional, melhor deverá ser o 
desempenho da empresa exportadora. Neste contexto, a indústria aeronáutica nacional possui extrema importância, porém, como o país é incapaz de absorver toda a produção, ela é exportada, marcando presença e tendo reconhecimento global de acordo com Fonseca et al (2017).

Segundo Root (1994), a maneira de entrada das empresas no mercado externo, podem ser classificadas em: Modo de exportação: exportação direta e exportação indireta; Modo de entradas contratuais: licenciamento, franchising, contrato de serviços e gerenciamento; Modo de investimentos: joint ventures e investimentos individuais.

\subsection{Problema em Estudo}

A Embraer é a terceira maior fabricante de aeronaves do mundo e através de toda sua cadeia produtiva é influenciada e impactada pela economia de inúmeros lugares, uma vez que a fabricação de aeronaves é dependente da importação de peças e serviços de países diferentes. (EMBRAER, 2018).

Essa desnacionalização da produção da Embraer acontece, principalmente, por conta da estratégia da empresa de inserção nos mercados internacionais de aviação e para ser melhor aceita pelas certificadoras estrangeiras. Sendo assim, é proposto pelos certificadores um conjunto de normas e diretrizes a serem seguidas por profissionais e agentes corporativos que tem como objetivo serem classificados como prestadores de serviços de excelência (TREVISAN, 2012).

Assim, a variação cambial é vista quando a empresa concretiza uma exportação e/ou importação com o pagamento para meses depois, a negociação do preço é feita em outra moeda, ficando sujeitas às variações do câmbio (BERNSTEIN, 1997).

\subsection{Objetivo do Trabalho}

Este trabalho tem como objetivo geral, analisar a influência da variação do câmbio na venda de aeronaves e na receita de uma empresa dependente do comércio internacional, a EMBRAER. O trabalho visa responder aos seguintes questionamentos:

i) Qual o impacto do dólar no volume de vendas de aeronaves da EMBRAER?

ii) Qual o impacto do dólar na receita total da EMBRAER?

Para o êxito desta pesquisa foram estabelecidos os seguintes parâmetros:

- Realizar uma revisão bibliográfica sobre os temas cadeia produtiva do setor aeronáutico e políticas cambiais;

- Realizar levantamento de dados referentes ao período de 2015 até 2018;

- Analisar os dados pesquisados através de modelo econométrico.

\subsection{Proposta Metodológica}

O trabalho foi desenvolvido em quatro pilares (FIGURA 1):

- Natureza: pesquisa aplicada,

- Abordagem: pesquisa quantitativa,

- Objetivo: pesquisa descritiva, e

- Procedimentos Técnicos: pesquisa bibliográfica e pesquisa documental. 


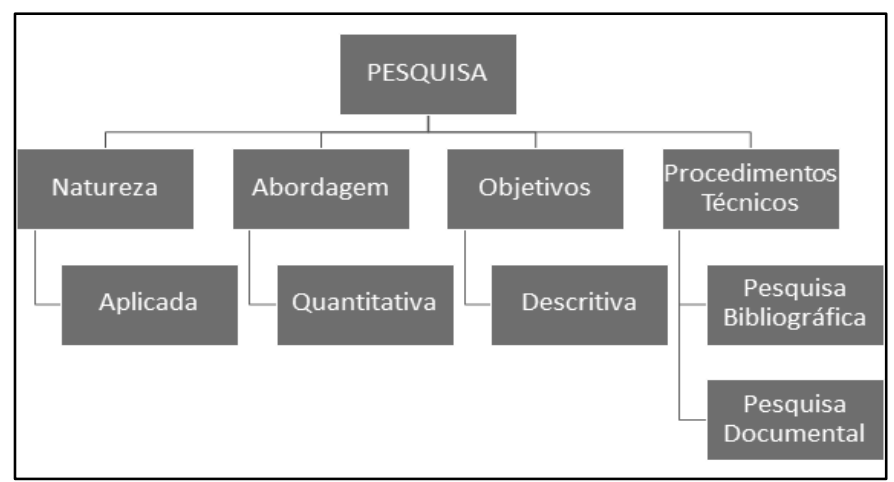

Figura 1. Proposta metodológica

\subsubsection{Natureza da Pesquisa}

Tem como escopo produção de conhecimentos com foco em solução de problemas específicos, com objetivo prático em verdades e interesses locais.

\subsubsection{Abordagem da Pesquisa}

Conforme Fonseca (2002) ao contrário da pesquisa qualitativa, os dados obtidos podem ser também quantificados. As amostras geralmente compreendem um sentido lato da população assim, os resultados são representação real de toda a população alvo da pesquisa. A pesquisa quantitativa representa um resultado de observação imparcial, considerando ainda a realidade com base de dados precisos, obtidos de forma técnica com instrumentos padronizados e neutros. A pesquisa também utiliza linguagem matemática para descrever as causas e demais variáveis de um fenômeno. Pode-se considerar o uso concomitante da pesquisa qualitativa e quantitativa que permitem recolher mais dados ao contrário do que no uso isolado das informações.

\subsubsection{Objetivos da Pesquisa}

A pesquisa descritiva exige um levantamento específico do que se deseja apurar. Esse tipo de proposta pretende estabelecer relações de variáveis de um certo fato ou acontecimento de determinada realidade (TRIVIÑOS, 1987). São exemplos disso: estudos de caso e pesquisa de opinião.

Ainda segundo Triviños, em razão da descrição exata de um fenômeno ou fato, pode haver críticas desses estudos, diferente do que verificado nas pesquisas de observação. Onde ainda para o autor, muita das vezes não existe por parte do pesquisador um exame crítico das informações, sendo muita das vezes equivocados onde as técnicas utilizadas podem, dependendo do tipo de apuração, haver imprecisão nos dados obtidos.

\subsubsection{Procedimentos Técnicos da Pesquisa}

A pesquisa bibliográfica utiliza fontes constituídas por material já elaborado, constituído basicamente por livros e artigos científicos localizados em bibliotecas. A pesquisa documental recorre a fontes mais diversificadas e dispersas, sem tratamento analítico, tais como: tabelas estatísticas, jornais, revistas, relatórios, documentos oficiais, cartas, filmes, fotografias, pinturas, tapeçarias, relatórios de empresas, vídeos de programas de televisão, etc. (FONSECA, 2002, p. 32).

A pesquisa documental por sua vez tem como base o levantamento de material teórico, já analisado e publicado por meio de qualquer fonte, sendo ela um livro, artigo científico, etc. Todo e qualquer trabalho científico é feita com base em pesquisas bibliográficas, que permitem ao pesquisador conhecer o que já foi estudado sobre determinado assunto. Existem também, pesquisas científicas que se baseiam exclusivamente em pesquisas bibliográficas, procurando referências teóricas publicadas com o intuito de obter informações ou conhecimentos anteriores ao problema a 
respeito do qual se procura resposta (FONSECA, 2002, p.32). Para Gil (2007, p. 44) os exemplos mais característicos desse tipo de pesquisa são sobre investigações e ideologias ou as que se propõem à análise das diversas posições do problema questionado.

\section{FUNDAMENTAÇÃO TEÓRICA}

Neste capítulo será apresentado os conceitos relevantes que compõem o trabalho de forma que foram abordados os seguintes temas:

- A cadeia produtiva aeronáutica;

- Políticas cambiais, flutuação e seus impactos na produção interna e;

- Cenário do setor aeronáutico nacional e políticas governamentais de apoio ao setor.

\subsection{Cadeia Produtiva Aeronáutica Brasileira}

De acordo com Ballou (2004) a cadeia de suprimentos é definida como um conjunto de atividades que se repetem inúmeras vezes ao longo da sequência pelo qual as matérias-primas serão convertidas em produtos acabados, com valor agregado ao consumidor. De forma genérica, a etapa de uma cadeia de abastecimento é composta por suprimentos, onde se encontram os fornecedores; produção, onde está a fabricação, estoque e embalagem; distribuição, onde ficam os produtos acabados e consumidores.

$\mathrm{Na}$ cadeia produtiva aeronáutica, uma das principais características é a modularidade dos subsistemas de montagens dos aviões. As empresas são divididas entre: produtoras de sistemas de propulsão, sistemas aviônicos e sistemas estruturais ou de fuselagem. São todas submetidas aos padrões de qualidade e fabricação da empresa líder que gerencia os projetos e integra os subsistemas fornecidos por empresas dos diferentes níveis (SALERNO, 2001).

A pirâmide exposta na Figura 2, evidencia os níveis de produção e as peças fabricadas em cada etapa da cadeia aeronáutica.

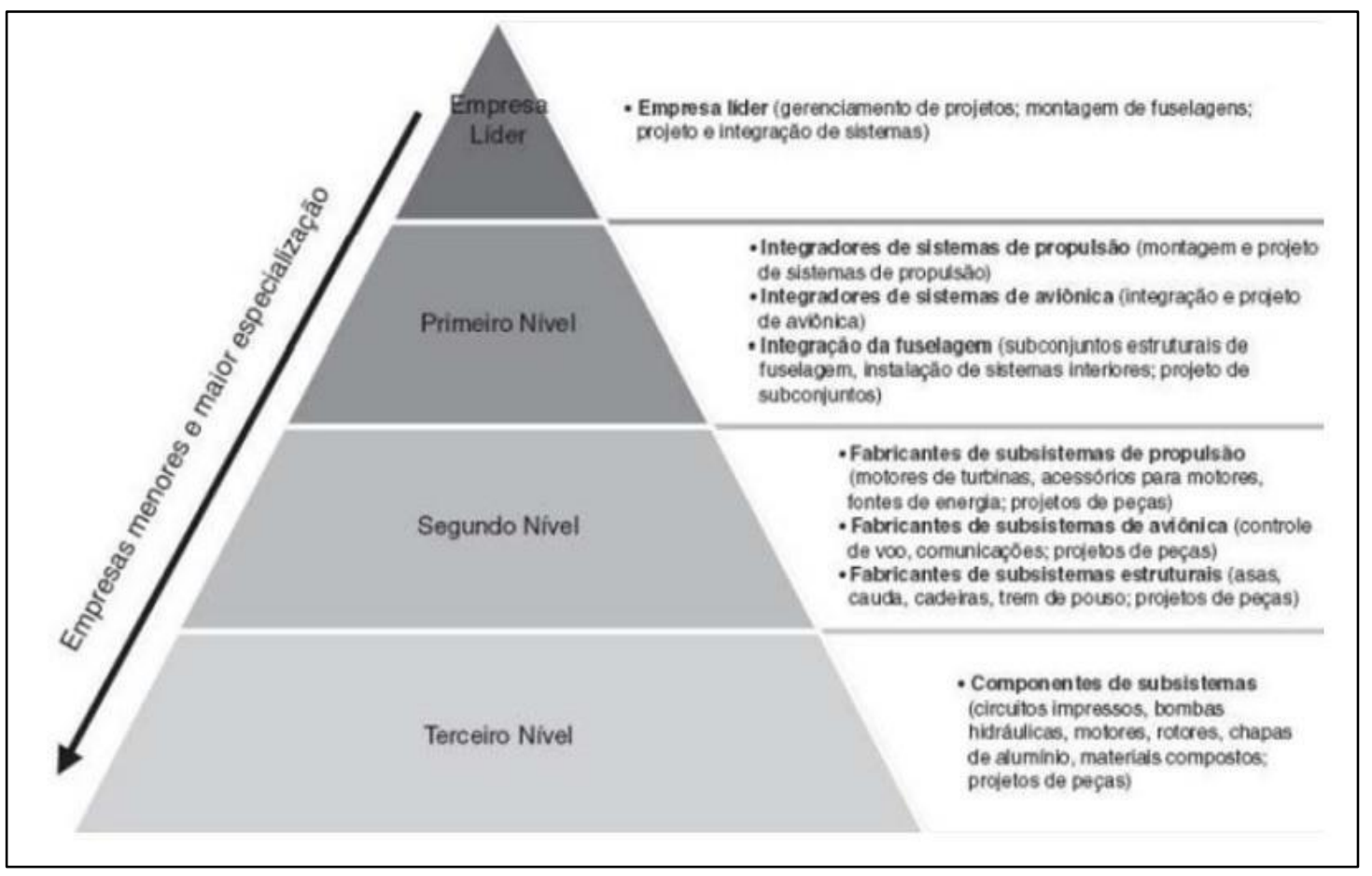




\section{Figura 2. Níveis de produção da cadeia aeronáutica}

Na pirâmide, é possível identificar como empresas líderes as integradoras de sistemas (Airbus, Boeing, Bombardier e Embraer). As empresas no primeiro nível são as fabricantes de sistemas complexos, composto pelos parceiros de risco que abastecem diretamente as empresas principais (empresas com altos custos de P\&D). No segundo nível estão as empresas fornecedoras de subsistemas complexos, que são insumos semiacabados, peças fundidas e usinagem que abastecem os fornecedores do primeiro nível (OLIVEIRA, 2005).

Um importante fator que justifica a concorrência desigual entre os fornecedores nacionais e internacionais é a elevada carga tributária brasileira, que apesar de recentes avanços, não livra inteiramente nas etapas seguintes os componentes que serão exportados quando incorporados ao produto final.

\subsection{Políticas Cambiais e Seus Impactos Na Produção Interna}

A internacionalização permite maior estabilidade pois as empresas se tornam menos dependentes do mercado interno, resultando na diversificação de suas operações e aumento da competitividade através da diluição dos custos fixos e redução dos custos médios, elevando assim a oferta de empregos qualificados, trazendo maior credibilidade ao exportador (RICHARDSON, 2001).

A partir de um modelo de equilíbrio geral, que visa explicar o comportamento da oferta, demanda e preços em uma economia que analisa mercados, Grimaldi (2011) investigou como nível de complexidade dos produtos exportados influenciam na economia nacional. Os resultados indicaram que quanto maior o nível de sofisticação da exportação, maior a taxa de crescimento da economia, a curto e longo prazo.

Conforme interpretado por Caparroz (2012), no que tange ao comércio internacional, na prática deixa claro o que um produto produzido com mais vantagens seria destinado à exportação, por outro lado, os produtos com menos vantagens e menor produção seriam importados de países com maior especialização.

Um importante fator que justifica a concorrência desigual entre os fornecedores nacionais e internacionais é a elevada carga tributária brasileira, que apesar de recentes avanços, não livra inteiramente nas etapas seguintes os componentes que serão exportados quando incorporados ao produto segundo Gomes et al. (2017).

De acordo com Bastos (2014), o regime de taxa de câmbio flutuante possui inúmeras vantagens, entre elas estão: equilíbrio nas contas externas, absorção de parte dos ajustes nas condições internas e externas, permite que a taxa de câmbio fique próxima do equilíbrio, pois é definida a partir de oferta e demanda. Porém, com este regime de câmbio flutuante, sua volatilidade é maior. Ainda baseado em Bastos, quando a moeda nacional fica desvalorizada, ocorre as pressões inflacionárias; quando ocorre a valorização da moeda, as exportações e indústrias nacionais são prejudicadas.

\subsection{Cenário Do Setor Aeronáutico e Políticas Nacionais De Apoio Ao Setor}

Conforme Gomes (2015), às agências de crédito à exportação (ECA) são atividades de Estado pertinentes a todos as economias mundiais, promovendo crédito as empresas com objetivo de geração de empregos e renda ao país proveniente. Assim, todos os países fabricantes de aeronaves, necessitam de apoio de uma ECA própria. Em 1997 o BNDES inicia suas operações de financiamento de aeronaves brasileiras para exportação. Sem esse apoio, a Embraer atuaria com desvantagem em comparação a canadense Bombardier, sua principal concorrente. 
De acordo Fonseca (2012) o BNDES firmou parceria com a Embraer no início da década de 1990, fato este que resultou num papel de extrema importância no sucesso da empresa que é visível hoje, conseguindo assim participação direta na venda de jatos e como consequência consolidando uma base forte para inserção no mercado internacional de aviação. Fonseca afirma ainda que esse importante apoio do BNDES também representou uma aliança entre o governo brasileiro com a empresa recém-privada. Sendo assim, essa parceria representou um grande salto no desenvolvimento tecnológico e industrial do país. A concentração desses recursos em uma única empresa pode ser explicada por uma aposta do BNDES à época na perspectiva de crescimento da Embraer e de valorização do capital da empresa na bolsa de valores, conjugada com a internacionalização da Embraer e por consequência compartilhar com esse novo mercado cultura, conhecimento, tecnologia e informação. Promovendo também a imagem do produto nacional. E para tanto, com parcerias com o Ministério do Desenvolvimento, Indústria e Comércio Exterior (MDIC), o governo brasileiro tem promovido feiras nacionais e internacionais, com o escopo de agregar valor econômico e incentivar pesadamente suas vendas externas. (FONSECA, 2012).

Para Gomes, Barcellos e Fonseca (2017), o apoio do BNDES como agência de crédito à exportação, tem sido imprescindível para garantir e ampliar as exportações de aeronaves.

De acordo Krugman e Obstfeld (2012), foi observado que uma empresa sempre aumenta suas vendas quanto maior for a demanda do seu produto, e o mesmo ocorre com o valor do produto em relação à quantidade de concorrentes. Também é verdade que uma empresa diminui as vendas quando há mais concorrência de outras empresas que ofertam o mesmo produto e reflete que, quanto maior o número de empresas, maior o seu custo médio, e que assim menor será a produção do produto ofertado.

Segundo Gomes (2015), as empresas líderes de mercado, também chamadas de players, operam com alto nível de concorrência e amplitude global.

A Figura 3 representa os concorrentes da Embraer:

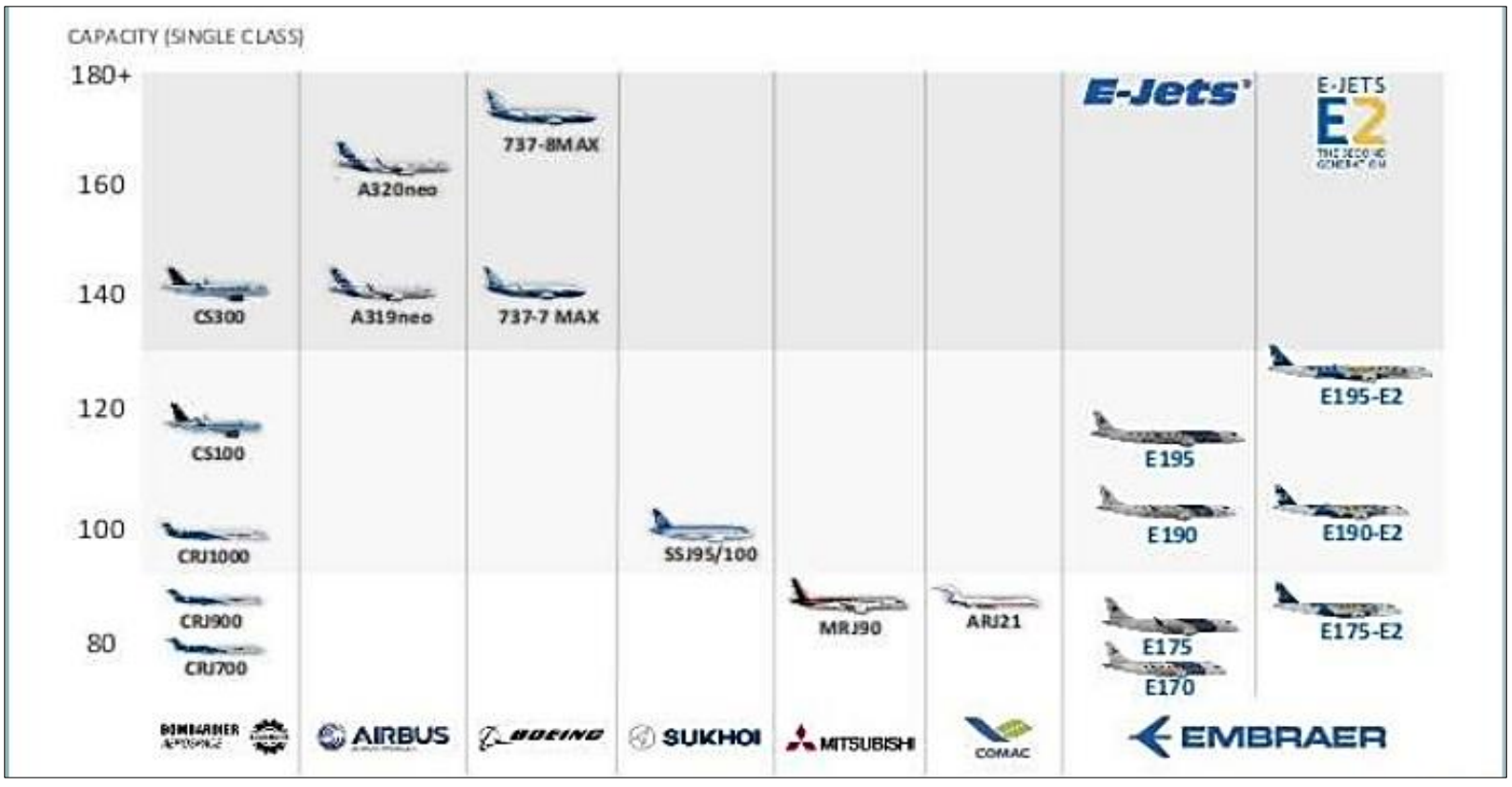

Figura 3. Cenário competitivo do setor Aeronáutico

Sendo assim Embraer tem como principal concorrente a empresa canadense Bombardier no setor de aviação regional com jatos médios de até 150 passageiros (GERVASONI, 2009). 
Para entrada no mercado chinês, a Embraer adotou pelo contrato de joint venture, que se é um acordo estratégico com um objetivo comercial comum e prazo determinado. Esse tipo de acordo visa unir os recursos das empresas para o desenvolvimento de um negócio conjunto e dividem os resultados. Em parceria com a Aviation Industry Corporation of China, foi criada em 2003 a Harbin Embraer, juntamente com mais duas subsidiárias estatais

De acordo com Santos et al. (2009), a internacionalização através do joint venture foi um fator determinante para expansão do mercado asiático, resultando vantagens a longo prazo. Após 13 anos, a parceria chegou ao fim em 2016.

\section{APRESENTAÇÃO DO CENÁRIO E ANÁLISE DOS MODELOS}

De acordo com Samuelson (1954) pode-se definir econometria como a análise quantitativa de eventos econômicos ocorridos, baseados na evolução da teoria juntamente com as observações utilizando métodos de interferências apropriados.

Conforme Wooldridge (2006) a utilização mais frequente da econometria é a previsão de variáveis macroeconômicas de grande relevância, como a taxa de juros, taxa de inflação e PIB (produto interno bruto).

Para Hoffman (2016) para ser entendida de forma pragmática e chegar a um consenso sem rodeios, precisamos entender os efeitos que algumas variáveis têm sobre outras, não obstante também às que somente parecem exercer, mesmo que não exista uma relação causal entre elas, podemos concatená-las por meio de expressões matemáticas e em consequência de isso estabelecer o valor de uma através de outras, todas sob determinadas condições.

A EMBRAER tem sua sede em São José dos Campos, cidade situada na região metropolitana do Vale do Paraíba que é um dos principais polos tecnológicos do Brasil. De acordo com dados coletados do informativo digital da empresa, foram elaborados os Gráficos 1 e 2. Conforme é demonstrado no Gráfico 1, nota-se que há uma certa estagnação na quantidade de aeronaves produzidas pela Embraer no período contemplado entre o primeiro trimestre de 2015 até o segundo trimestre de 2018. Essa conclusão é confirmada pela linha pontilhada que demonstra a tendência de fabricação ao longo do tempo.

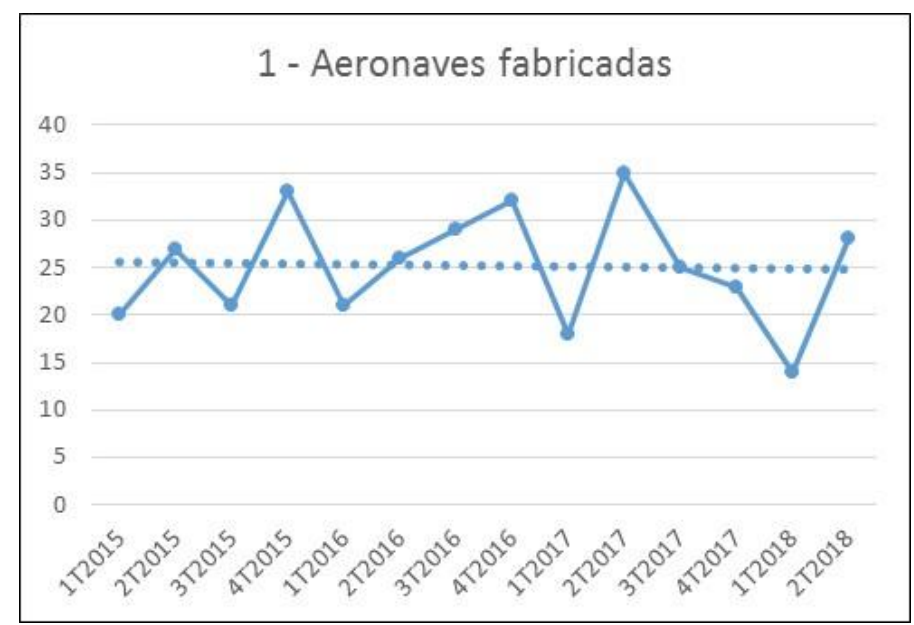

Gráfico 1. Aeronaves fabricadas por trimestre

No Gráfico 2, é possível observar que existe grande variação da receita liquida ao longo do período analisado, e que esta variação segue uma linha de tendência decrescente. 


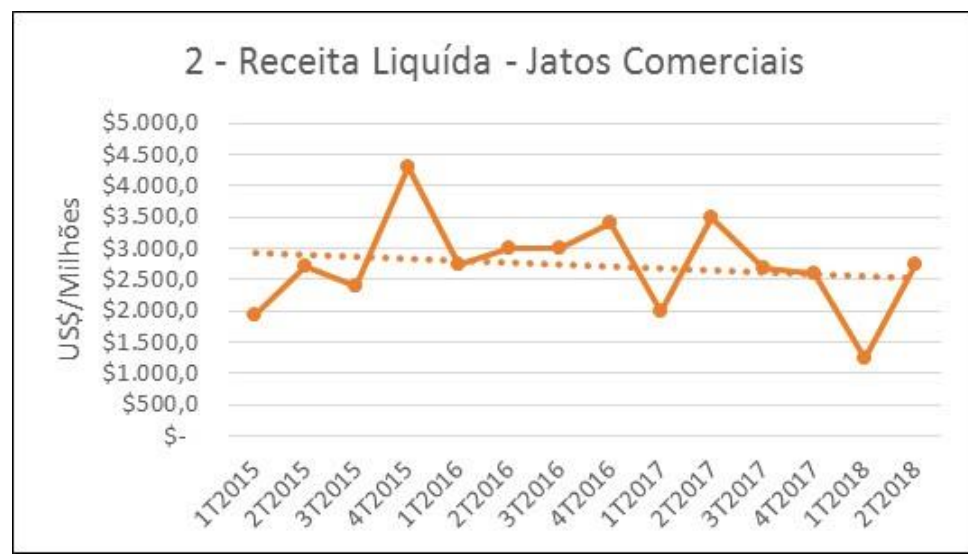

Gráfico 2. Receita liquida da venda de jatos por trimestre.

Segundo Vieira (2016), a regressão é utilizada para descrever que um modelo matemático foi adaptado aos dados para explicar a variação de uma variável $Y$ em função da variação da variável $X$, cuja influência é representada pelo $\mathrm{R}^{2}$, que é o coeficiente de determinação. Foram desenvolvidos, em Excel, dois modelos de regressão linear para avaliar a possível relação e influência entre os dados coletados.

O Modelo 1 analisa a relação entre a receita liquida da empresa e a variação cambial. É possível analisar que o R-quadrado (coeficiente de determinação) possuí valor muito baixo, ou seja, apenas $8.14 \%$ da variação da receita liquida é explicada pela variação cambial.

\begin{tabular}{|c|l|r|l|}
\hline R-quadrado & \multicolumn{2}{|c|}{0.0814772569002144} & \\
\hline F de significação & \multicolumn{2}{|c|}{0.322547454} & valor-P \\
\hline \multirow{2}{*}{ Coeficientes } & Interseção & 310.3617365 & 0.897349152 \\
\cline { 2 - 4 } & Taxa de câmbio - R\$ / US\$ & 712.2700247 & 0.322547454 \\
\hline
\end{tabular}

O Modelo 2 foi desenvolvido a partir da relação entre a quantidade de aeronaves vendidas e a receita liquida. Com um R-quadrado de $83.71 \%$, é possível entender a grande influência entre os dados. Esse modelo, apresenta como resultado também, que cada aeronave vendida, resulta em um lucro líquido de aproximadamente $\mathrm{R} \$ 112.000 .000$ reais para a empresa.

\begin{tabular}{|l|l|l|l|}
\hline R-quadrado & \multicolumn{2}{|c|}{0.83712996560037} & \\
\hline F de significação & \multicolumn{1}{|c|}{$4.54042925536104 \mathrm{E}-06$} & valor-P \\
\hline \multirow{2}{*}{ Coeficientes } & Interseção & -97.9838 & 0.795552856 \\
\cline { 2 - 4 } & Aeronaves vendidas & 112.5482 & 0.000004540 \\
\hline
\end{tabular}

Baseado nos resultados encontrados e a partir da análise dos mesmos, é possível inferir que o câmbio não é uma variável considerável diretamente para a receita líquida, entretanto há correlação positiva entre a receita liquida e a quantidade de vendas de aeronaves comerciais. Outras variáveis não contempladas neste estudo podem demonstrar maior correlação entre si.

\section{CONSIDERAÇÕES FINAIS}

O presente estudo teve como objetivo analisar a influência da flutuação do câmbio nas vendas de aeronaves, estudando especificamente dados referentes a EMBRAER. Ressalta-se que os dados coletados são de domínio público e acessados por meio dos relatórios divulgados trimestralmente pela própria empresa. 
Os dados coletados referem-se ao período de 2015 até 2018 de forma trimestral. O desenvolvimento do modelo matemático permitiu analisar o efeito da flutuação cambial na venda de aeronaves. Percebe-se que não há impacto considerável no período estudado.

É interessante, porém, notar que toda a pesquisa bibliográfica aponta a relevância da flutuação cambial. Esse fato pode ser justificado pelo período em análise. Houve muita variação do câmbio nos anos pesquisados. Entende-se que essa flutuação é consequência das crises política e econômica ocorridas no país.

Recomenda-se, que se continue o levantamento dos dados para estimar novos modelos e gerar comparações com àqueles obtidos no presente trabalho.

\section{REFERÊNCIAS}

BALLOU, R. H. Gerenciamento da cadeia de suprimentos/Logística Empresarial. 5. ed. Porto Alegre: Bookman, 2004.

BASTOS, E. K. X.; Fonte, P. V. S. Mercado de câmbio Brasileiro, intervenções do Banco Central e controles de capitais de 1999 a 2012. Instituto de Pesquisa Econômica Aplicada (IPEA), No. 1934. Brasília. 2014

BERNSTEIN, P. L. Desafio aos Deuses. São Paulo: Campus, 1997.

CAPARROZ, R. Comércio Internacional Esquematizado. São Paulo: Saraiva, 2012.

EMBRAER, Relatórios anuais. Disponível em: https://ri.embraer.com.br/show.aspx?idCanal=FQxR65+1OmnFsUzhIziJFQ== . Acesso em 20/04/2018.

FONSECA, J. J. S. Metodologia da pesquisa científica. Fortaleza: UEC, 2002.

FONSECA, Paulus Vinícius da Rocha. Embraer: um caso de sucesso com apoio do BNDES. Revista do BNDES, jun. 2012.

FONSECA, M.S. Parque tecnológico de santa maria-rs: do sonho à (dura) realidade. 2017. Dissertação (Programa De Pós-Graduação Em Desenvolvimento Regional Mestrado E Doutorado) Universidade de Santa Cruz do Sul, Santa Cruz do Sul, 2017.

GERVASONI, Viviane. Resenha: Internacionalização e os países emergentes. Revista Gestão \&amp; Regionalidade, São Paulo, n. 73, v. 25, p. 113-114, jan./abr. 2009.

GIANNETTI, R. O paradoxo cambial e as exportações brasileiras. Global. Out. 2005

GIL, A. C. Métodos e técnicas de pesquisa social. 5.ed. São Paulo: Atlas, 2007.

GOMES, S. B. V.; NOVIS FILHO, S. A. Garantia incondicional ou seguro de crédito à exportação de aeronaves: Visões da experiência internacional. BNDES Setorial, Rio de Janeiro, n. 42 p. 5-54, set. 2015 .

GOMES, S. B. V. et al. O apoio ao desenvolvimento do setor de aeroespaço e defesa: visões da experiência internacional. BNDES Setorial, Rio de Janeiro, n. 45, p.7-55, mar. 2017.

GRIMALDI, Daniel Da Silva. Padrão de sofisticação exportador e crescimento na economia brasileira. 2011. Dissertação (Mestrado em Economia no curso de Economia da Faculdade de Economia, Administração e Contabilidade) - USP - Universidade de São Paulo, São Paulo, 2011. 
HOFFMANN, R. Análise de regressão: uma introdução à econometria [recurso eletrônico] / Rodolfo Hoffmann. - - Piracicaba: ESALQ/USP, 2016. 393 p. : il.

KRUGMAN, P.; OBSTFELD, M. International Economics: Theory and policy. Pearson. Boston, 9. ed. 2012

OLIVEIRA, L.G. A Cadeia De Produção Aeronáutica No Brasil: uma análise sobre os fornecedores da Embraer. 2005. 226 p. Tese (Doutorado Em Política Científica E Tecnológica) Instituto De Geociências, Universidade Estadual De Campinas, Campinas, 2005.

RICHARDSON, J. David. Exports Matter... And so Does Trade Finance. In: HUFBAUER, Gary C.; RODRIGUEZ, Rita M. The Ex-Im Bank in the 21st Century: A New Approach? Washington, DC: Peterson Institute for International Economics, 2001.

ROOT, F. R. Entry Strategies for International Markets. New York: Lexington Books, 1994.

SALERNO, M. S. The characteristics and the role of modularity in the automotive business. International Journal Automotive Technology and Management, v. 1, n. 1, p. 92-107, 2001

SAMUELSON, P.A.; KOOPMANS, T. C.; STONE, J. R. N. Report of he evaluative committee for econometrica. Econommétrica. Abr. 1954, v. 22, n. 2, p. 141-146

SANTOS, Geraldo Claro et al. Estudo das Estratégias de Internacionalização da EMBRAER: O desafio da competitividade sustentada no setor aeronáutico. In: ENCONTRO LATINO AMERICANO DE INICIAÇÃO CIENTÍFICA E ENCONTRO LATINO AMERICANO DE PÓSGRADUAÇÃO, 13; 9., 2009

TREVISAN, L.; BERNARDES, A. A moderna logística no território brasileiro: contribuição à pesquisa. Ciência Geográfica - Bauru - XVI - Vol. XVI - (2): Jan.-Dez. 2012.

TRIVINÕS, A. N. S. Introdução à pesquisa em ciências sociais: a pesquisa qualitativa em educação. São Paulo: Atlas, 1987

VIEIRA, Sonia. Introdução a Bioestatistica. 5. ed. Rio de Janeiro: Elsevier, 2016. 246 p. v. 1.

WILLIAMSON, J. Exchange rate policy and development, presented in Initiative for Policy Dialogue Task Force on Macroeconomics. Nova York, 2003.

WOOLDRIDGE, J. M. Introdução à Econometria: uma abordagem moderna. São Paulo: Thomson Pioneira, 2006. 